University of Wollongong

Research Online

Australian Institute for Innovative Materials -

Papers

Australian Institute for Innovative Materials

$1-1-2015$

Efficient, absorption-powered artificial muscles based on carbon nanotube hybrid yarns

\author{
Marcio D. Lima \\ University of Texas \\ Mohammad W. Hussain \\ University of Texas at Dallas \\ Geoffrey M. Spinks \\ University of Wollongong, gspinks@uow.edu.au \\ Sina Naficy \\ University of Wollongong, snaficy@uow.edu.au \\ Daniela Hagenasr \\ University of Texas
}

See next page for additional authors

Follow this and additional works at: https://ro.uow.edu.au/aiimpapers

Part of the Engineering Commons, and the Physical Sciences and Mathematics Commons

Research Online is the open access institutional repository for the University of Wollongong. For further information contact the UOW Library: research-pubs@uow.edu.au 


\title{
Efficient, absorption-powered artificial muscles based on carbon nanotube hybrid yarns
}

\author{
Abstract \\ A new type of absorption-powered artificial muscle provides high performance without needing a \\ temperature change. These muscles, comprising coiled carbon nanotube fibers infiltrated with silicone \\ rubber, can contract up to $50 \%$ to generate up to $1.2 \mathrm{~kJ} \mathrm{~kg}^{-1}$. The drive mechanism for actuation is the \\ rubber swelling during exposure to a nonpolar solvent. Theoretical energy efficiency conversion can be as \\ high as $16 \%$.

\section{Keywords} \\ powered, nanotube, yarns, artificial, efficient, hybrid, absorption, muscles, carbon \\ Disciplines \\ Engineering | Physical Sciences and Mathematics

\section{Publication Details} \\ Lima, M. Dias., Hussain, M. W., Spinks, G. M., Naficy, S., Hagenasr, D., Bykova, J. S., Tolly, D. \& Baughman, \\ R. H. (2015). Efficient, absorption-powered artificial muscles based on carbon nanotube hybrid yarns. \\ Small, 11 (26), 3113-3118.
}

\section{Authors}

Marcio D. Lima, Mohammad W. Hussain, Geoffrey M. Spinks, Sina Naficy, Daniela Hagenasr, Julia S. Bykova, Derrick Tolly, and Ray H. Baughman 
DOI: $10.1002 /(($ please add manuscript number $))$

Article type: Communication

\section{Efficient, Absorption-Powered Artificial Muscles Based on Carbon Nanotube Hybrid Yarns}

Márcio Dias Lima, Mohammad W. Hussain, Geoffrey M. Spinks, Sina Naficy, Daniela Hagenasr, Julia S. Bykova, Derrick Tolly, and Ray H. Baughman*

Dr. M. D. Lima, M. W. Hussain, D. Hagenasr, Dr. J. S. Bykova, D. Tolly, Prof. R.

H. Baughman

The Alan G MacDiarmid NanoTech Institute

The University of Texas at Dallas

800 West Campbell Road BE26

Richardson, Texas 75080, USA

E-mail: ray.baughman@utdallas.edu

Dr. S. Naficy, Prof. G. M. Spinks

School of Mechanical, Materials and Mechatronic Engineering, Intelligent Polymer Research Institute, Australian Research Council Centre of Excellence for Electromaterials Science University of Wollongong

Northfields Avenue, Wollongong, NSW 2522, Australia

D. Tolly

LINTEC Nano-Science and Technology Center

990 N. Bowser Road, Suite 800

Richardson, Texas 75081, USA

Keywords: artificial muscle, carbon nanotube yarn, absorption-powered muscle, coiled muscle, tensile actuator

Thermally driven, twisted carbon nanotube yarns and twisted high strength polymer fibers generate impressive tensile actuation, providing large strokes and vastly exceeding the work and power capabilities of natural skeletal muscle. However, operating temperatures can be high when giant strokes are required and energy conversion efficiencies are presently below $2 \%$. We here report chemically powered artificial muscles that provide high performance without requiring a temperature change. These muscles, comprising twisted and coiled carbon nanotube fiber infiltrated with silicone rubber, can contract up to $50 \%$, generate up to $1.2 \mathrm{~kJ} \mathrm{~kg}^{-1}\left(1 \mathrm{MJ} \mathrm{m}^{3}\right)$ of mechanical energy and support up to $45 \mathrm{MPa}$ of mechanical load. The drive mechanism for actuation is rubber swelling during exposure to a non-polar solvent. During solvent absorption, which can be completed in less than $0.25 \mathrm{sec}$, the helical 
structure of the coiled fibers converts the expansion of rubber inside the yarn into contraction along the coil axis, thereby allowing a full cycle of actuation at $1 \mathrm{~Hz}$, while a $25 \mu \mathrm{m}$ thick and $20 \mathrm{~mm}$ long coiled fiber lifts a 23,000 times heavier load than it's liquid-free weight. This new actuation mechanism also exhibits a natural catch state, so energy is not required to retain actuator stroke. Scalability of muscle yarns over a 20 fold diameter range is demonstrated. The theoretical conversion efficiency between chemical and mechanical energy is estimated, based on experimental results and the initial and final states of the system, to be as high as $16 \%$.

Coiled carbon nanotube (CNT) yarns and polymer fibers have been used as reversible, large stroke, high power and high work-capacity artificial muscles. ${ }^{[1,2,3]}$ The nanoscale helical topology of these systems enables conversion of yarn or fiber volume change to torsional actuation, which drives large-stroke tensile actuation of coiled muscles. Such mechanism for thermally or electrothermally driven actuators can generate strokes of 5-10\% and a contractile work capacity of $1.36 \mathrm{~kJ} \mathrm{~kg}^{-1}$ for paraffin-wax-filled CNT yarns ${ }^{[1]}$ and above $30 \%$ stroke and $2.48 \mathrm{~kJ} \mathrm{~kg}^{-1}$ contractile work capacity for highly twisted nylon-6,6 fibers. ${ }^{[3]}$ These work capacities greatly exceed that generated by natural skeletal muscle $\left(39 \mathrm{~J} \mathrm{~kg}^{-1}\right),{ }^{[4]}$ but large temperature changes are needed to produce giant strokes and the efficiency of thermal to mechanical energy conversion is presently low $(<2 \%)$.

Chemically powered artificial muscles offer potential solutions to these two problems, although fuel-powered actuators have not yet provided performance that matches or exceeds in all aspects the performance of natural muscle. The gravimetric energy density of liquid fuel greatly exceeds that of batteries, meaning that direct conversion of chemical energy to mechanical energy is the preferred mechanism for mobile systems. While combustion engines involve major heat generation, natural muscle efficiently converts chemical energy to mechanical power with only minor temperature rise (about $40 \%$ efficiency). ${ }^{[5]}$ Isothermal polymer actuators using chemical species to change volume can mimic natural muscle 
function. Expanding gels that respond to changes in $\mathrm{pH}$ or ionic strength can generate very large strokes, but have low load supporting capabilities and are slow to respond unless diffusion distances are engineered to be short. ${ }^{[6]}$ Systems utilizing dimensional changes produced by absorption and desorption of swelling solvents have been used to produce linear, bending ${ }^{[7]}$ or torsional actuation. ${ }^{[8]}$ Fast response can be achieved using highly porous materials ${ }^{[9]}$ or very thin structures. ${ }^{[8]}$ Fuel-powered muscles that utilize the chemical energy of combustion, rather than the much smaller energy of solvent-induced swelling, have been demonstrated, but either the muscle stroke was small or actuation was by thermo-chemical processes, which restricted the realizable energy conversion efficiencies. ${ }^{[10]}$

We here describe a new type of solvent-driven actuator that uses a helically twisted nanofiber yarn to convert the volumetric expansion of a contained guest material into a large tensile contraction, thereby providing strokes and contractile work capacities exceeding natural muscle. These materials exploit the same mechanism first shown for coiled carbon nanotubes yarns filled with paraffin wax ${ }^{[1]}$ and later for highly twisted polymeric fibers. ${ }^{[3]}$ Volume expansion of a guest material within a CNT yarn or of a twisted polymer fiber causes reversible untwist, as first demonstrated for electrochemically-driven neat CNT yarns. ${ }^{[11]}$ Due to the geometry of the coiled yarn or fiber, the torque generated by yarn or fiber untwist acts to pull adjacent coils together, providing the ability to do work by exploiting contraction along the coil axis. In previous examples, the volume change of the working material was primarily driven by thermal expansion. We here explore solvent sorption and desorption that allows nearly isothermal operation. Since our system also responds thermally, so we are able to compare the efficiency of conversion of heat or chemical energy to mechanical energy in the same system.

A cross-linked elastomer was chosen as the solvent exchanging guest material in the hybrid CNT yarn muscles. In contact with a solvent, some cross-linked elastomers can provide volumetric swelling of up to $400 \%,{ }^{[12]}$ which is considerably larger than the $\sim 30 \%$ 
volume increase when paraffin wax is heated to $200^{\circ} \mathrm{C}$. Equilibrium swelling in elastomers is reached when there is a balance between the reduction of free energy due to the mixing of the cross-linked polymer and the solvent and the elastic energy necessary to deform (expand) the polymer. The mixing free energy of a solvent-polymer pair is characterized by the FloryHuggins Chi parameter $(\chi)$ and we have chosen a range of solvents with different $\chi$ for the silicone rubber guest material, with $\chi$ determined from free swelling experiments (Supporting Information).

Hybrid carbon nanotubes yarn was produced by infiltrating a slightly twisted yarn of highly oriented CNT aerogel sheets with uncured silicone rubber before coiling of the yarn. After polymerization of the rubber, the resulting CNT-silicone composite was twisted to produce a coiled yarn. The coils were obtained by overtwisting CNT-silicone hybrid yarns under a constant load, as described previously. ${ }^{[1,3]}$ Infiltrating the CNTs sheets before twist and coiling results in a structure with significantly more guest material (silicone rubber) than for paraffin-wax-filled CNT yarns. A final hybrid yarn, containing 90 to $95 \mathrm{wt} \%$ silicone rubber, is shown in Figure 1b. Contraction experiments were conducted either by dropwise application of solvent directly to the yarn or by exposing the coiled yarn to solvent vapor inside a capillary tube, as shown in Figure 1a. For all experiments, a uniformly coiled hybrid muscle was tethered at opposite ends to prohibit torsional rotation of the coiled yarn.

Exposure of the hybrid coiled yarns to swelling solvents induced large tensile contractions. The axial coil contraction is a direct result of yarn untwist due to solvent absorption. To conserve total twist, yarn untwist is converted to coil up-twist (increased writhe) to provide contraction, without yielding any torsional actuation of the coiled yarn. A more detailed analysis of the actuation mechanism can be found in the supporting information.

As shown in Figure 1c, tensile actuator strokes of nearly 50\% can be achieved in the coils. By exposing the material to an air flow or simply letting the solvent evaporate, the concentration of solvent inside the coiled yarn decreases and the coil elongates to its original 
length. This highly reversible actuation by solvent absorption/evaporation was stable over a thousand of cycles (Figure S1).
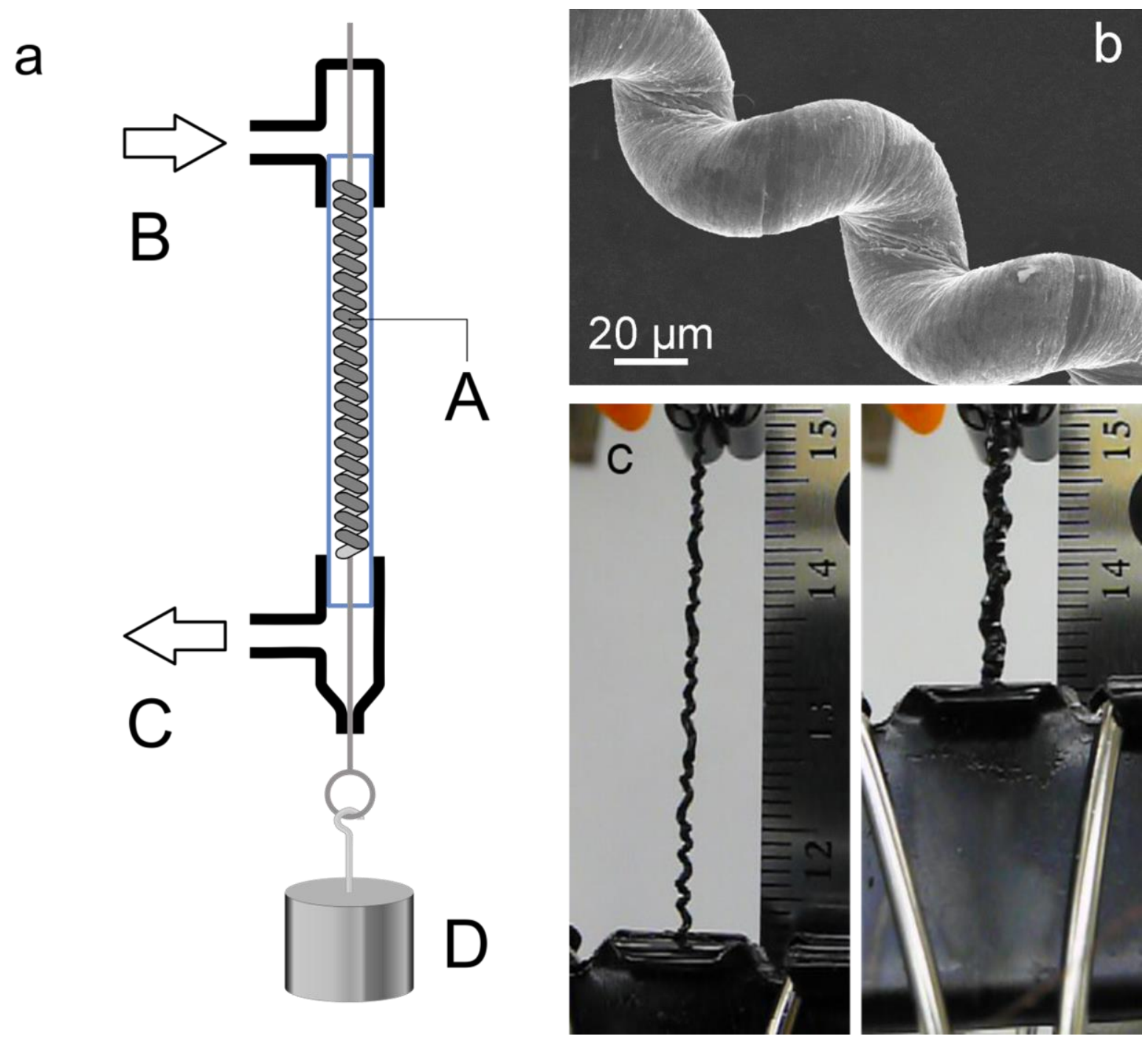

Figure 1. a) Setup used for measurement of chemically-induced actuation: the hybrid yarn (A) is enclosed inside a capillary tube having an inlet (B) and outlet (C) for solvent and gas flow, and a load (D) is applied to the hybrid muscle; b) SEM of a thin coiled silicone-CNT hybrid yarn; c) photographs showing $50 \%$ contraction of a $500 \mu \mathrm{m}$ thick coiled yarn when exposed to hexane under $2 \mathrm{MPa}$ tensile stress. Here and elsewhere tensile stresses are with respect to the non-coiled diameter of hybrid yarn before solvent absorption.

It is interesting to compare chemical actuation of the coiled hybrid silicone-CNT yarn with that for a fiber of neat silicone rubber. Even though CNTs are a minor component of the hybrid actuator (less than $10 \mathrm{wt} \%$ ) they have a dramatic effect on the mechanical response during solvent absorption and evaporation. When exposed to hexane, a fiber of pure silicone rubber (under 0.3 MPa tensile stress) expands in both diameter and length, providing a tensile 
stroke of $\sim 4 \%$ (Figure S2). In contrast, the helically aligned network of CNTs in the coiled silicone-CNT hybrid yarn converts the solvent-induced rubber volume increase into a contraction, which is an order of magnitude higher and of opposite sign to the tensile stroke of the neat silicone rubber. The CNTs also mechanically stiffen the rubber and increase actuation force and work capacities. A silicone-CNT hybrid coiled actuator generated $\sim 50 \%$ contraction while lifting a load that is 23,000 times heavier than the actuators liquid-free weight, while the neat silicone rubber lifted only 42 times its own weight (when both had a fiber diameter-tolength ratio of 80). For additional comparisons, water responsive gels and acetone responsive sponges were reported to lift 380 times $^{[13]}$ and 25 times $^{[9]}$ their own liquid-free weights, respectively.

We can also compare chemical and electrothermal actuation for the same coiled silicone-CNT hybrid yarn. In both cases rubber expansion leads to yarn untwist and muscle contraction. Reflecting the high loading of silicon rubber, electrothermally powered contractions up to $33 \%$ were demonstrated (Figure 2a), which is significantly higher than reported for previous carbon nanotube based hybrid yarn actuators. ${ }^{[1,14]}$ Solvent expansion using hexane produced actuation $\sim 5$ times more slowly (Figure 2b) than was achieved by rapid electrothermal heating of the same yarn, but produced a larger contraction (46\%). By reducing the diameter of the yarn, both chemical and thermal actuations can be accelerated, as shown in Figures 2c and 2d. A 20-fold increase in diameter caused a 42.5 times increase in contraction time and a 42 times increase in expansion time. Due to the characteristics of the actuator fabrication process, the change in diameter also produced a variation in the CNTs concentration from $9.5 \mathrm{wt} \%$. (Figure $2 \mathrm{~d}$ ) in the $25 \mu \mathrm{m}$ thick yarn to $5 \%$ for the $500 \mu \mathrm{m}$ yarn (Fig 2c). Note in Table S2 that the progressively decreasing weight percent CNTs in the silicone/CNT hybrid yarn with increasing fiber diameter caused the muscle contraction to progressively increase from $15 \%$ for $9.5 \mathrm{wt} \%$ CNTs in the $25 \mu \mathrm{m}$ diameter yarn to $50 \%$ for the $5 \mathrm{wt} \% \mathrm{CNT}$ in the $500 \mu \mathrm{m}$ diameter yarn. This result suggests the major effect of the 
CNTs on mechanically limiting volume expansion during solvent sorption, and thereby

limiting tensile contraction. However, reduction of the wt\% CNTs reduces the tensile stress that can be applied while achieving highly reversible actuation.

Contraction times as short as $0.1 \mathrm{sec}$ were measured for solvent-induced yarn actuation (Figure 2d and Movie S1), which allowed the muscle coil to operate at $1 \mathrm{~Hz}$ (Movie S2) to achieve $\sim 2.5 \%$ stroke. A similar stroke could be achieved electro-thermally at $10 \mathrm{~Hz}$

(Figure 2c), with resonance effects pushing the stroke to $\sim 7 \%$ after the initial cycles.
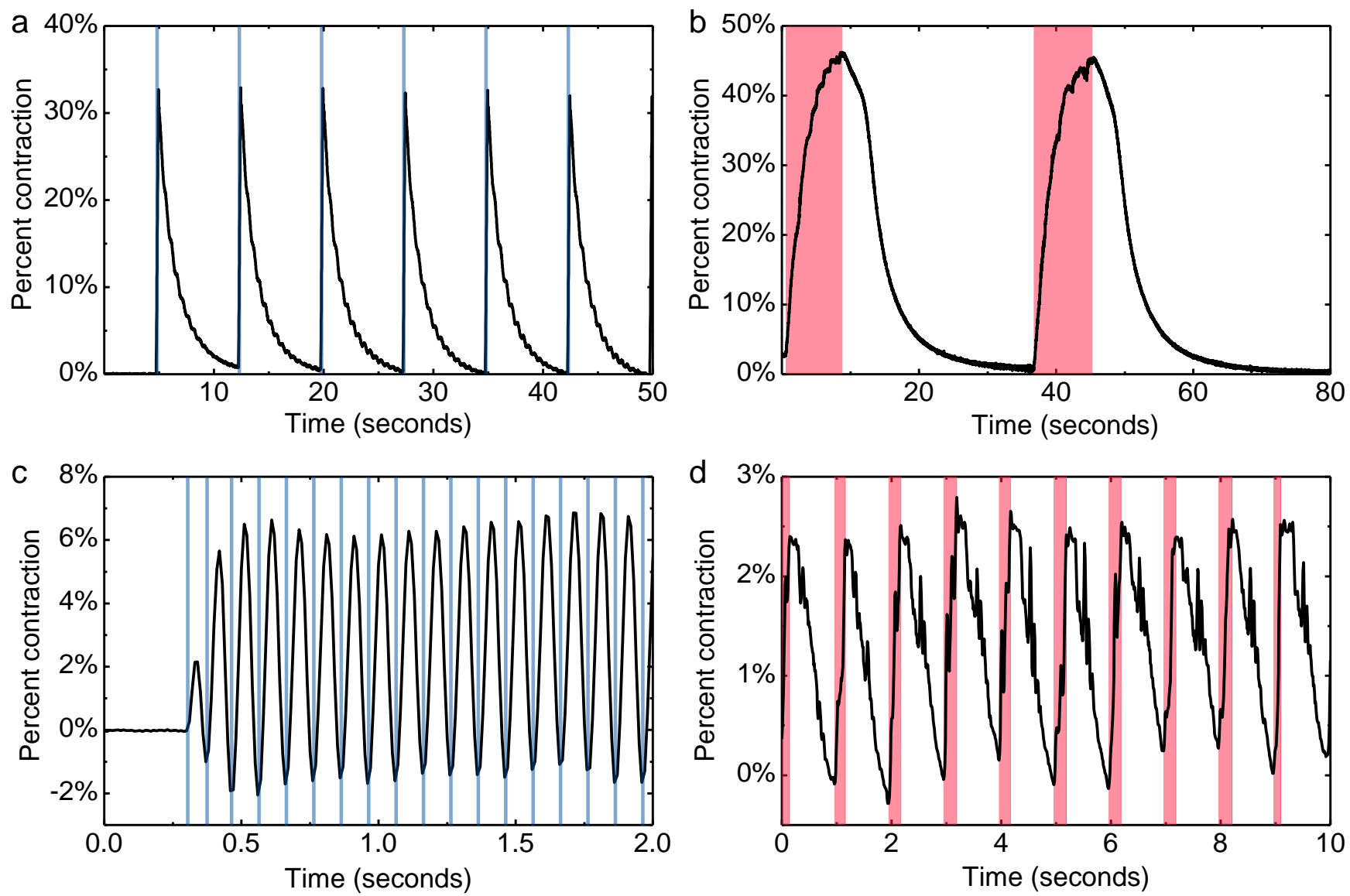

Figure 2. a) Electrically driven actuation of a coiled $500 \mu \mathrm{m}$ diameter silicone-CNT hybrid yarn under 1.5 MPa tensile stress when driven by $50 \mathrm{~ms}$ long square-wave electrical pulses (blue lines) at $0.133 \mathrm{~Hz}$ and b) the chemical actuation of an identical yarn under the same load when exposed to hexane for approximately 8 seconds (pink areas), after which the hexane was allowed to evaporate in still air. c) Electrothermal actuation of 20-mm-long, coiled $25 \mu \mathrm{m}$ diameter yarn muscle during $10 \mathrm{~Hz}$ pulses (blue) under $14 \mathrm{MPa}$ stress and d) the same yarn during chemical actuation by hexane at $1 \mathrm{~Hz}$ under the same mechanical load, where the width of the red line represents the time period of solvent exposure. 
Figure 3a shows the load dependence of the specific work accomplished during contraction for a coiled silicone-CNT hybrid yarn as a function of the applied tensile stress. We here define specific work as the amount of work (in $\mathrm{J} \mathrm{kg}^{-1}$ ) done by the muscle in lifting a weight during solvent-induced contraction, divided by the mass of the solvent free muscle. At an optimal stress at $20 \mathrm{MPa}$ the maximum specific work was $1180 \mathrm{~J} \mathrm{~kg}^{-1}$. This work capacity is much higher than the $76 \mathrm{~J} \mathrm{~kg}^{-1}$ reported for previous solvent-driven actuators. ${ }^{[13]}$

The contraction of the coiled fibers strongly depends on the solvent used to drive actuation. As expected, a strong correlation exists between coil contraction and the degree of swelling of the neat silicone rubber (Figure 3b). The small swelling caused by high polarity solvents like ethanol caused almost no actuation. Less polar solvents like chloroform, ether and hexane produce large swelling and higher actuation strokes. The large difference in swelling and actuation between solvents affords a different actuation method, where cycles of contraction and expansion can be achieved by alternating between miscible high and low swelling solvents. We call this solvent-switch actuation, as compared to single-solvent actuation, where actuation is reversed by solvent evaporation.

Figure 3c shows tensile actuation that is produced by alternately exposing the coiled hybrid fiber to ethanol and ethyl ether. Very rapid response now occurs in both contraction and expansion directions, in contrast to the relatively slow expansion due to solvent evaporation. Ethyl ether is highly soluble in ethanol and a flow of ethanol quickly removes it from the polymeric matrix of the coiled yarn, resulting in rapid expansion of the coiled yarn muscle. Contraction occurred in less than $0.25 \mathrm{sec}$, with the actuator producing $1.1 \mathrm{~kJ} \mathrm{~kg}^{-1}$ of specific work and $4.4 \mathrm{~kW} \mathrm{~kg}^{-1}$ of mechanical power during contraction, which is much higher than provided by a moisture driven torsional actuator $\left(71.9 \mathrm{~W} \mathrm{~kg}^{-1}\right)^{[8]}$ or a moisture driven bending actuator $\left(2.5 \mathrm{~W} \mathrm{~kg}^{-1}\right){ }^{[13]}$

A further advantage of this solvent exchange system is a 'catch state'. When immersed in either solvent the actuator maintains its length without requiring any external source of 
energy. This effect can be extremely useful for practical applications, such as the control valve shown in Figure 3d. A chemically selective valve is demonstrated that automatically routes the liquid stream according to its polarity. The low polarity, high swelling solvent closes the valve and directs flow from inlet $\mathrm{A}$ to outlet $\mathrm{B}$, while a high polarity low swelling solvent opens the valve so liquid exits from outlet $\mathrm{C}$. With reaction times as fast as 0.25 seconds, this valve shows higher speeds than that for previously reported chemical valves. For instance, $\mathrm{pH}$ sensitive chemical valves have been reported that provide reaction times between 6 and 8 seconds. ${ }^{[15]}$

The energy conversion efficiency of these high-stroke, high-power artificial muscles is a key practical consideration, especially for portable applications. We next calculate, using experimental data, the theoretical maximum energy conversion efficiency for chemicallydriven coiled silicone-CNT hybrid muscles. The input energy is the energy necessary to return the system (coiled actuator and solvents) to its initial state after one contraction/expansion cycle. Since contraction and expansion of the coil are spontaneous processes for both singlesolvent and solvent-switch actuation, the only stage of the actuation cycle that requires energy is recycling of the solvents. Recycling involves condensation of the solvent vapor removed by evaporation for single-solvent actuation and solvent separation (for instance, by osmosis) for solvent-switch actuation. Therefore, the relevant energies are the enthalpy of evaporation for the former and the enthalpy of dissolution for the latter. For condensation of the solvent vapor, it is necessary to extract heat from the solvent vapor and that can be achieved with an efficiency defined by the Coefficient of Performance (COP, ratio of cooling provided to energy consumed). For liquid-gas single solvent actuation using hexane, a reduction in temperature from 25 to $-10^{\circ} \mathrm{C}$ would drop the hexane vapor pressure from 180 to 25 torr, thereby recovering $86 \%$ of the hexane from the gas stream (that can be recycled), giving a maximum $C O P$ of $6.5\left(C O P_{\text {cooling }}=T_{c} /\left(T_{h}-T_{c}\right)\right.$, where $T_{c}$ and $T_{h}$ are the temperatures of the cold and hot sides, respectively). Measurements of the change in diameter of the coiled muscle 
fiber during absorption of hexane during muscle contraction (Figure S3b) shows a yarn diameter increase from 58 to $75 \mu \mathrm{m}$, corresponding to a 1.67 times increase in volume due to hexane absorption and $0.43 \mathrm{~g}$ of added hexane for each gram of hybrid yarn. Assuming that this is the total amount of solvent used per cycle and using a $C O P$ of 6.5 , the latent heat of condensation of hexane $\left(335 \mathrm{~J} \mathrm{~g}^{-1}\right)$, and the $93 \mathrm{~J}$ needed for cooling down each gram of hexane and the gas in which it is diluted (assuming it is air which is initially saturated with vapor), it will take $65 \mathrm{~J} \mathrm{~g}^{-1}$ to recover the hexane. Using the specific work ( $\left.1.2 \mathrm{~J} \mathrm{~g}^{-1}\right)$ measured during contraction of the hybrid yarn, the estimated efficiency of chemical energy to mechanical energy conversion during contraction is therefore $4.3 \%$.

For the case of solvent-switch actuation, energy is needed to separate the solvents after expansion of the coiled fiber. From Figure $3 \mathrm{c}$ we obtain that the specific work done on contraction (using ethyl ether and ethanol) is $1180 \mathrm{~kJ} \mathrm{~kg}^{-1}$. The hybrid coiled yarn volume increases approximately 1.6 times during ethyl ether absorption. That results in $0.42 \mathrm{~g}$ of ether for each gram of dry silicone-CNT hybrid yarn. Since separation from ethanol and ether requires at least $16 \mathrm{~J} \mathrm{~g}^{-1}$ (Figure $\mathrm{S3a}$ ), in the worst case where the ethyl ether is very diluted in ethanol and assuming a $100 \%$ efficient separation process the efficiency from chemical to mechanical energy conversion is $16 \%$. Both efficiencies are superior to that for previous CNT-based electrothermally driven muscles $(\sim 0.5 \%){ }^{[1]}$

While not yet optimized, these solvent driven artificial muscles already demonstrate stroke, force generation, and work capabilities that are superior to natural skeletal muscle. Using a non-polar, high-swelling solvent, the silicone rubber infused CNT yarns generated $50 \%$ contraction and $1180 \mathrm{~J} \mathrm{~kg}^{-1}$ work during contraction (compared with $20 \%$ and $39 \mathrm{~J} \mathrm{~kg}^{-1}$, respectively for natural muscle). ${ }^{[5]}$ Contraction times of 0.25 seconds, obtained when using solvent-switch actuation, are comparable to contraction times in natural muscle. These performances for the artificial muscles yield an estimated theoretical energy conversion efficiency of $16 \%$, based on the measured mechanical work output. However, we have not yet 
explored other solvent-rubber systems. The maximum volumetric swelling of the silicone rubber used here was $167 \%$, which is well below that for other systems, which can be as high as $400 \%{ }^{[12]}$ Such high swelling is achieved in rubbers of lower cross-link density and it remains to be demonstrated whether such systems can generate sufficient swelling pressure to induce large actuation in the coiled CNT yarn.

Using appropriate pumps and valves, the hybrid yarn muscles could be used for various applications, such as for robotics, prosthetics and medical devices. Especially interestingly, two waste streams from a chemical plant (containing yarn expanding and yarn contracting chemicals, respectively) could be applied alternatively to the tensile actuators to generate mechanical energy that can be harvested as electrical energy (by using yarn tensile contraction and subsequent expansion under a returning force to rotate a rotational electromagnetic energy generator in alternatively forward and reverse directions). If the absorbed waste stream is hotter than the non-absorptive waste steam, sorptive and thermal actuation could be effectively combined to increase electrical energy output per cycle. Additionally, the present tensile actuation could be used to open and close valves, depending upon the concentration of a sorptive liquid to a liquid flow. While the cost of CNT sheets and yarns is presently prohibitive for applications that require massive muscle weights, the approach described is applicable for other host nanofiber or microfiber sheets and yarns, like those obtainable by inserting twist and coiling in guest-coated, electrospun, oriented polymer nanofiber sheets. 

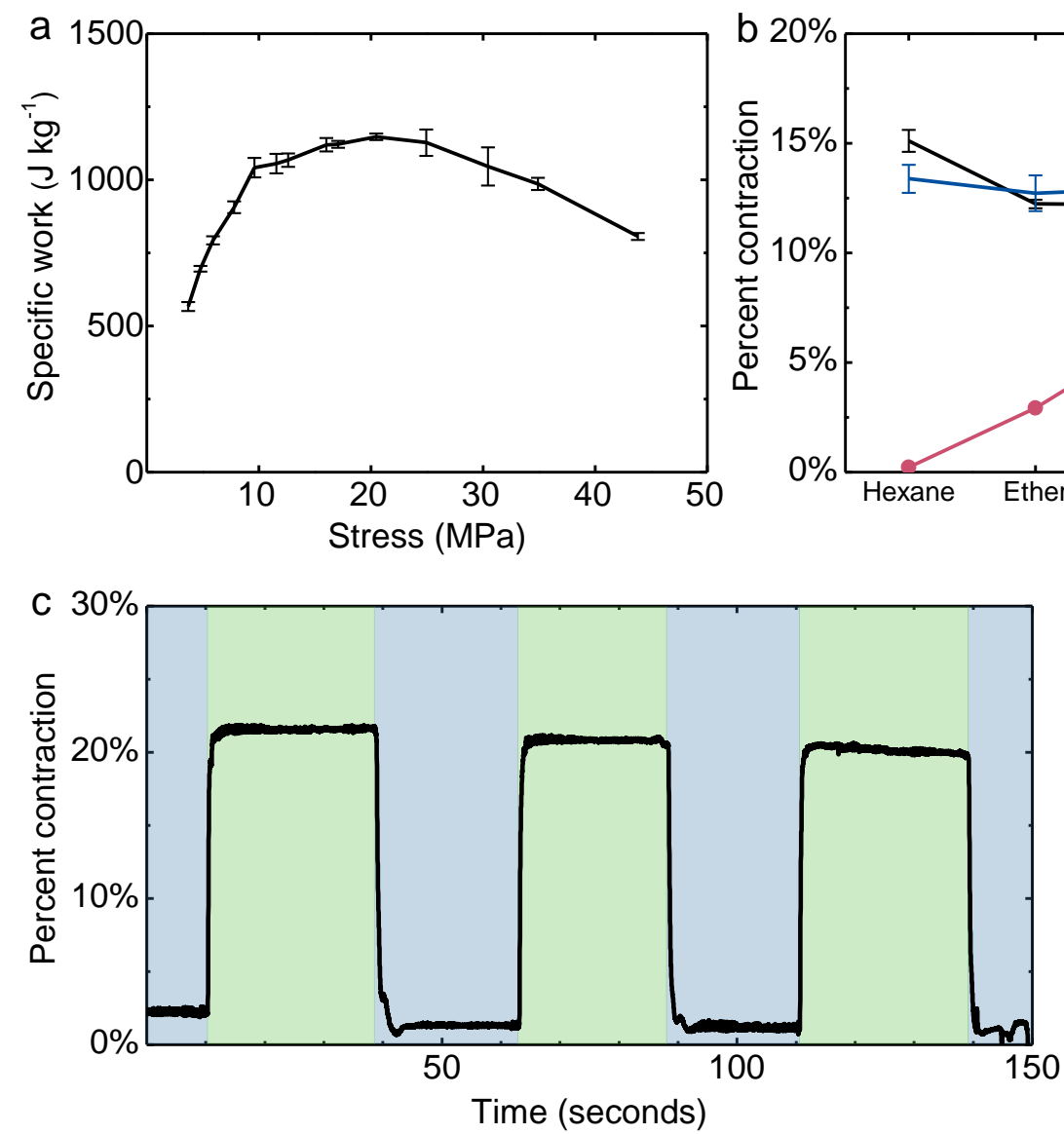

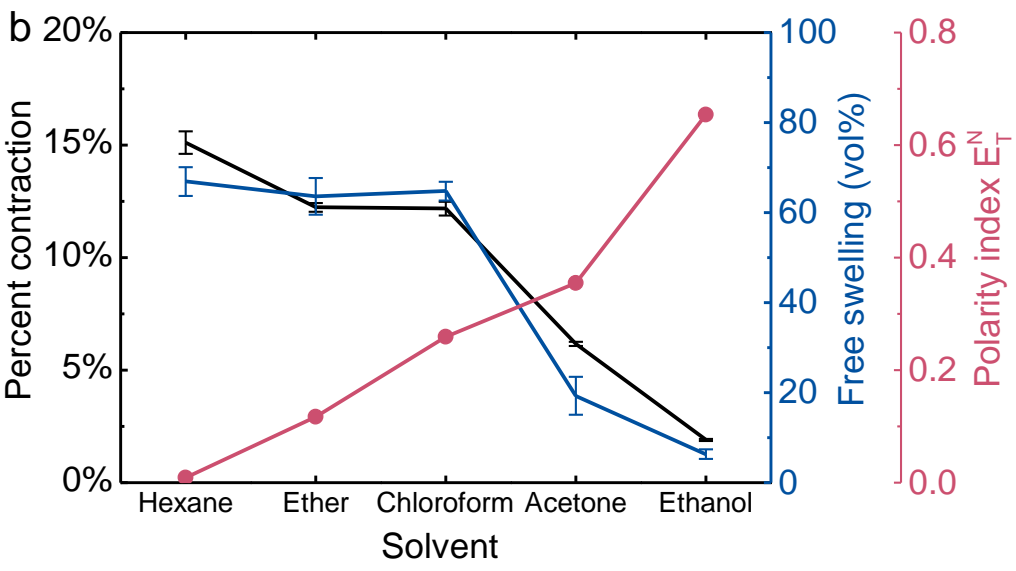

d
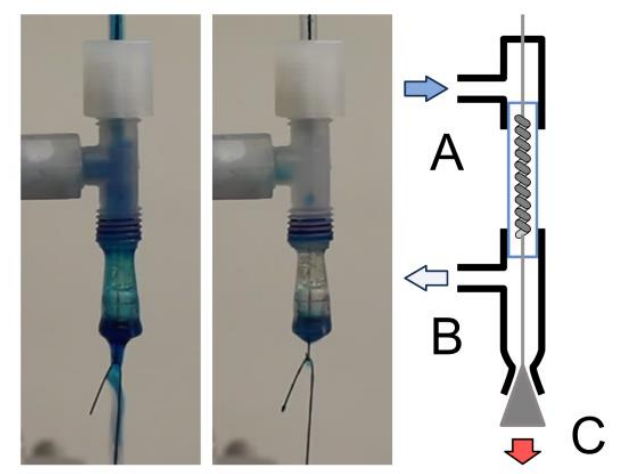

Figure 3. a) The load dependence of the specific work during contraction of a coiled siliconeCNT hybrid yarn muscle due to absorption of hexane; b) comparison of actuation driven by different solvents for a coiled yarn under 5.3 MPa tensile stress. Solvent polarity indexes (red triangles) and the percent swelling of neat silicone rubber in the solvents (blue circles) are given on the right. c) Actuation of a coiled hybrid yarn exposed to two solvents with different polarities: ethanol (blue) and ethyl ether (green) under 11.6 MPa of tensile stress.

d) A chemically selective valve which routes a fluid according to its polarity. When ethanol (dyed blue) is flowing through the valve with inlet A, the coil remains extended, making the flow exit through the bottom path $\mathrm{C}$. When ethyl ether flows through the valve it causes contraction of the coiled muscle, closing the bottom exit $\mathrm{C}$ and forcing the liquid to flow through the lateral exit B. The diameters of the yarns in the coiled fiber muscles in the experiments of a), b), and c) are 35,45 , and $40 \mu \mathrm{m}$, respectively.

\section{Experimental Section}

Synthesis of carbon nanotubes. Carbon multiwall nanotube (MWNT) forests, that were

drawable to produce MWNT aerogel sheets, were grown by chemical vapor deposition (CVD) using acetylene $\left(\mathrm{C}_{2} \mathrm{H}_{2}\right)$ gas as the carbon precursor. Silicon wafers coated with a 1-3 nm thick iron catalyst layer by e-beam evaporation were used as substrates. For MWNT growth, the substrates were loaded into a 3.5-inch diameter quartz tube furnace that had been heated to $700^{\circ} \mathrm{C}$ in a mixture of $500 \mathrm{sccm} \mathrm{Ar}$ and $75 \mathrm{sccm} \mathrm{H}$. After 5 minutes of residence time at oven 
temperature, the nanotube forests were grown during 2 to 5 minutes after the introduction of $50 \mathrm{sccm}$ of $\mathrm{C}_{2} \mathrm{H}_{2}$ to this gas mixture. The used MWNT forests were typically about $350 \mu \mathrm{m}$ high and the nanotubes had average outer diameters of $\sim 9 \mathrm{~nm}$ and contained $\sim 6$ walls. Transparent, highly oriented MWNT sheets are fabricated by solid-state draw from the above MWNT forests, as described elsewhere. ${ }^{[16]}$

Fabrication of silicone-CNT hybrid yarn. A $2.0 \mathrm{~cm}$ wide sheet carbon nanotube aerogel sheet was pulled from a carbon nanotube forest to about $20 \mathrm{~cm}$ length. Such sheets were stacked until the amount of CNT required for a targeted yarn diameter was obtained. Using a low degree of twist insertion, the sheet stack was then twisted into a low density yarn by using an electric motor to provide a yarn bias angle (the angle on fiber surface between yarn axis and nanotube orientation) of $5^{\circ}$ to $10^{\circ}$, which is not enough to fully collapse the CNT aerogel. The amount of infiltrated silicone resin was controlled by the degree of twist in the yarn at the moment of the infiltration. Highly twisted yarns have less internal void space between the nanotubes before infiltration and will absorb less silicone resin. Once the neat yarn was uniformly twisted to low bias angle, a coating of silicone was applied to the yarn using a rod covered with the uncured silicone. The used silicone rubber resin is Mold Max 25 from Smooth-On, Inc., which was delivered to the low twist yarn while diluted with hexane (2:1 by weight). After 24 hour curing at room temperature, the carbon nanotube yarn infiltrated with silicone was then re-attached to the motor used for twist insertion and twisted until it was fully coiled. The required amount of additional twist to produce complete coiling reduced by onethird the length of the cured silicone/CNT yarn. Cross-sectional images at low and highresolution (Figure S4) of an infiltrated yarn cut by a focused ion beam (which causes minimal damage to the yarn structure) confirms that that the silicone rubber has been uniformly infiltrated into the yarn actuator. The concentration of CNTs was calculated by measuring the total area of CNT sheets used on the yarn fabrication (areal density of the CNT sheets: $3 \mu \mathrm{g} \mathrm{cm}^{-2}$ ) and the mass of the hybrid CNT-silicone yarn after coiling. The final coiled muscle 
yarns are then attached to an apparatus shown in Figure 1a for characterization of actuation. Actuator stroke was optimized by adjusting the amount of twist in the coiled fiber until the desired maximum contraction was achieved for a given applied load.

Characterization. Displacements during actuation were measured using a contactless inductive proximity sensor (Omega LD701 5/10) with data acquisition (Omega module OMUSB-1408Fs). A Motic digital optical microscope from Microscope World was used to measure the expansion of the coiled hybrid yarns when exposed to hexane. A Zeiss-LEO Model 1530 scanning electron microscope was used for imaging the hybrid yarns. Weight calculations done by optical microscopy were calibrated against directly measured weights. The values agreed within $8 \%$.

\section{Supporting Information}

Supporting Information is available from the Wiley Online Library or from the author.

\section{Acknowledgements}

Supported by Air Force Office of Scientific Research grant FA9550-12-1-0211 and Robert A. Welch Foundation grant AT-0029.

Received: ((will be filled in by the editorial staff))

Revised: ((will be filled in by the editorial staff)) Published online: ((will be filled in by the editorial staff))

[1] M. D. Lima, N. Li, M. J. de Andrade, S. Fang, J. Oh, G. M. Spinks, M. E. Kozlov, C.

S. Haines, D. Suh, J. Foroughi, S. J. Kim, Y. Chen, T. Ware, M. K. Shin, L. D. Machado, A. F. Fonseca, J. D. W. Madden, W. E. Voit, D. S. Galvão, R. H. Baughman, Science. 2012, 338, 928.

[2] R. H. Baughman, C. Cui, A. A. Zakhidov, Z. Iqbal, J. N. Barisci, G. M. Spinks, G. G. Wallace, A. Mazzoldi, D. De Rossi, A. G. Rinzler, O. Jaschinski, S. Roth, M. Kertesz, Science. 1999, 284, 1340. 
[3] C. S. Haines, M. D. Lima, N. Li , G. M. Spinks, J. Foroughi, J. D. W. Madden, S. H.

Kim, S. Fang, M. J. de Andrade, F. Göktepe, Ö. Göktepe, S. M. Mirvakili, S. Naficy, X.

Lepró, J. Oh, M. E. Kozlov, S. J. Kim, X. Xu, B. J. Swedlove, G. G. Wallace, R. H.

Baughman, Science. 2014, 343, 868.

[4] R. K. Josephson, Annu. Rev. Physiol. 1993, 55, 527.

[5] J. D. W. Madden, N. A. Vandesteeg, P. A. Anquetil, P. G. A. Madden, A. Takshi, R. Z. Pytel, S. R. Lafontaine, P. A. Wieringa, I. W. Hunter, IEEE J. Oceanic Eng. 2004, 29, 706.

[6] A. Sidorenko, T. Krupenkin, A. Taylor, P. Fratzl, J. Aizenberg, Science. 2007, 315, 487.

[7] J. Lu, S.-G. Kim, S. Lee, I.-K. Oh, Adv. Funct. Mater. 2008, 18, 1290.

[8] H. Cheng, Y. Hu, F. Zhao, Z. Dong, Y. Wang, N. Chen, Z. Zhang, L. Qu, Adv. Mater. 2014, 26, 2909.

[9] Q. Zhao, J. W. C. Dunlop, X. Qiu, F. Huang, Z. Zhang, J. Heyda, J. Dzubiella, M. Antonietti, J. Yuan, Nat. Commun. 2014, 5, 4293.

[10] V. H. Ebron, Z. Yang, D. J. Seyer, M. Kozlov, J. Oh, H. Xie, J. Razal, L. J. Hall, J. P. Ferraris, A. G. MacDiarmid, R.H. Baughman, Science. 2006, 311, 1580.

[11] J. Foroughi, G. M. Spinks, G. G. Wallace, J. Oh, M. E. Kozlov, S. Fang, T. Mirfakhrai, J. D. W. Madden, M. K. Shin, S. J. Kim, R. H. Baughman, Science. 2011, 334, 494.

[12] Shin-Etsu Chemical Co., Ltd. 2005. Characteristic properties of Silicone Rubber Compounds. Last modified June, 2012. http://www.silicone.jp/e/catalog/pdf/rubber_e.pdf.

[13] M. Ma, L. Guo, D. G. Anderson, R. Langer, Science. 2013, 339, 186.

[14] a) J. A. Lee, Y. T. Kim, G. M. Spinks, D. Suh, X. Lepró, M. D. Lima, R. H.

Baughman, S. J. Kim, Nano Lett. 2014, 14, 2664; b) K.-Y. Chun, S. H. Kim, M. K. Shin, C. H.

Kwon, J. Park, Y. T. Kim, G. M. Spinks, M. D. Lima, C. S. Haines, R. H. Baughman, S. J.

Kim, Nat. Commun. 2014, 5, 3322. 
[15] a) B. Zhao, J. S. Moore, Langmuir. 2001, 17, 4758; b) D. J. Beebe, J. S. Moore, J. M.

Bauer, Q. Yu, R. H. Liu, C. Devadoss, B.-H. Jo, Nature. 2000, 404, 588.

[16] X. Lepró, M. D. Lima, R. H. Baughman, Carbon. 2010, 48, 3621. 
A new type of absorption-powered artificial muscles provide high performance without needed temperature change. These muscles, comprising coiled carbon nanotube fiber infiltrated with silicone rubber, can contract up to $50 \%$ to generate up to $1.2 \mathrm{~kJ} \mathrm{~kg}^{-1}$. The drive mechanism for actuation is rubber swelling during exposure to a non-polar solvent.

Theoretical energy efficiency conversion can be as high as $16 \%$.

\section{Keyword}

artificial muscle, carbon nanotube yarn, absorption-powered muscle, coiled muscle, tensile actuator

M. D. Lima, M. W. Hussain, G. M. Spinks, S. Naficy, D. Hagenasr, J. S. Bykova, D. Tolly, R. H. Baughman*

\section{Efficient, Absorption-Powered Artificial Muscles Based on Carbon Nanotube Hybrid} Yarns
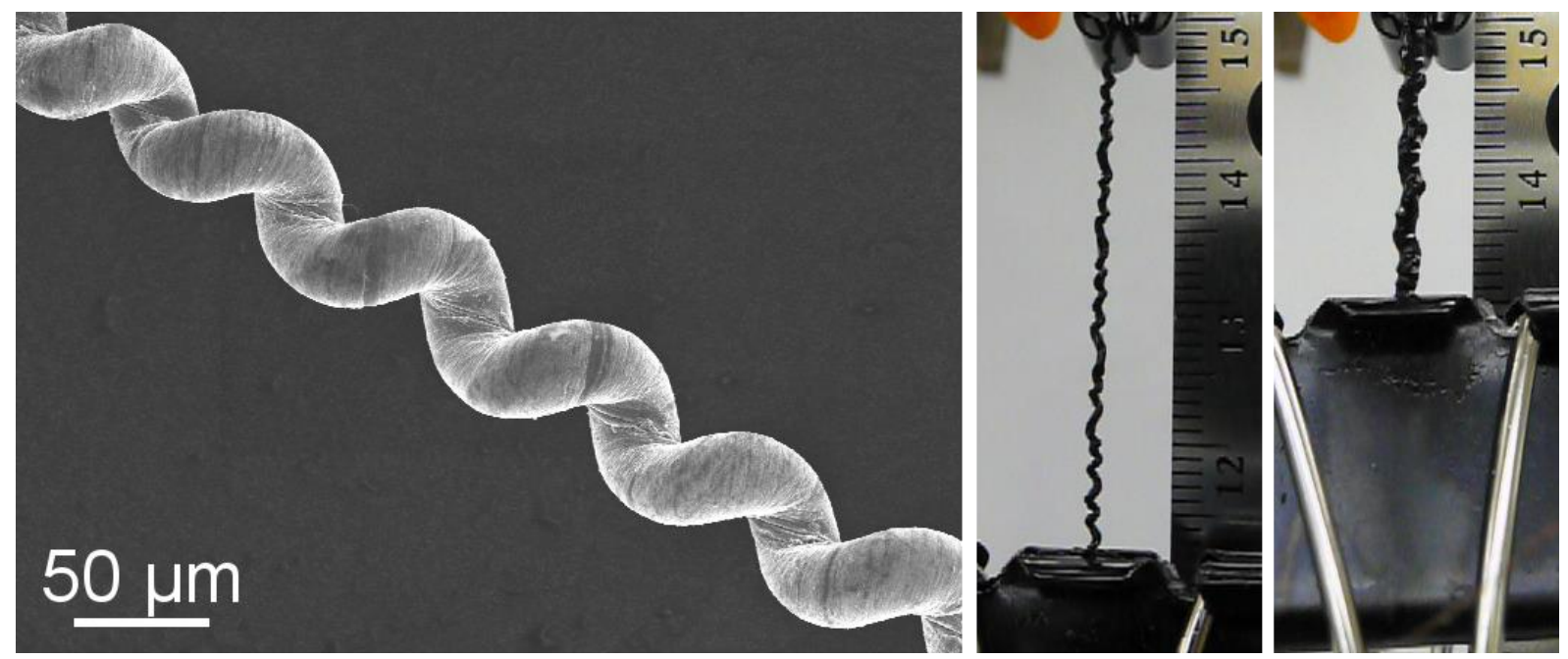